\title{
Comparison of Framingham and REGICOR scales for calculating cardiovascular risk in a cohort of patients with morbid obesity
}

FJ Vílchez-López, Isabel Mateo-Gavira, María Belén Ojeda-Schuldt, Luis Escobar-Jiménez, Manuel Aguilar-Diosdado

Endocrinology and Nutrition Department, Puerta del Mar Hospital, Cádiz, Spain.

\section{INTRODUCTION}

$\checkmark$ The prevalence of obesity has increased, reaching epidemic proportions. Framingham and REGICOR scales predict the likelihood of developing a coronary event in the next 10 years.

\section{OBJECTIVES}

$\checkmark$ The aim of this study was to assess the concordance of these scales in the stratification of cardiovascular risk (CVR) in a cohort of patients with morbid obesity

\section{METHODS}

$\checkmark$ Cross-sectional study including a sample of patients with morbid obesity evaluated from 2005 to 2012.

$\checkmark$ The estimation of CVR at 10 years was determined according to the Framingham-Wilson Score and its adaptation to Mediterranean populations (REGICOR scale). These scales include the following variables: age, sex, total cholesterol levels, HDL cholesterol, systolic blood pressure, presence of diabetes (T2DM) and smoking.

$\checkmark$ Depending on the results, they were classified into 3 categories: low risk (<10\%), intermediate (10-20\%) and high (> $20 \%)$.

\section{RESULTS}

$\checkmark$ A total of 211 patients were included. The mean age was $37.9 \pm 9.8$ years and 157 were female (74\%). The mean BMI was $51.13 \pm 7.11 \mathrm{~kg} / \mathrm{m} 2$. Regarding cardiovascular risk factors, 75 (35.7\%) patients had hypertension, 75 (35.7\%) dyslipidemia, 62 (29.5\%) smoking and 53 (25.2\%) T2DM. Total cholesterol mean levels were $197.27 \pm 38.85 \mathrm{mg} / \mathrm{dl}$ and HDL $44.19 \pm 11.53 \mathrm{mg} / \mathrm{dl}$

$\checkmark$ The results for the estimation of CVR with the REGICOR scale were: $93.9 \%$ of patients with low risk, $5 \%$ intermediate and $1 \%$ high risk, while those with the Framingham score were: $77.2 \%$ low risk, $13.9 \%$ intermediate and $8.9 \%$ high. .

$\checkmark$ The mean CVR estimation was $3.08 \pm 2.85$ with REGICOR versus $6.16 \pm 7.02 \%$ with Framingham ( $p<0.001$ ). The Kappa index of agreement between the scales was 0.231 .

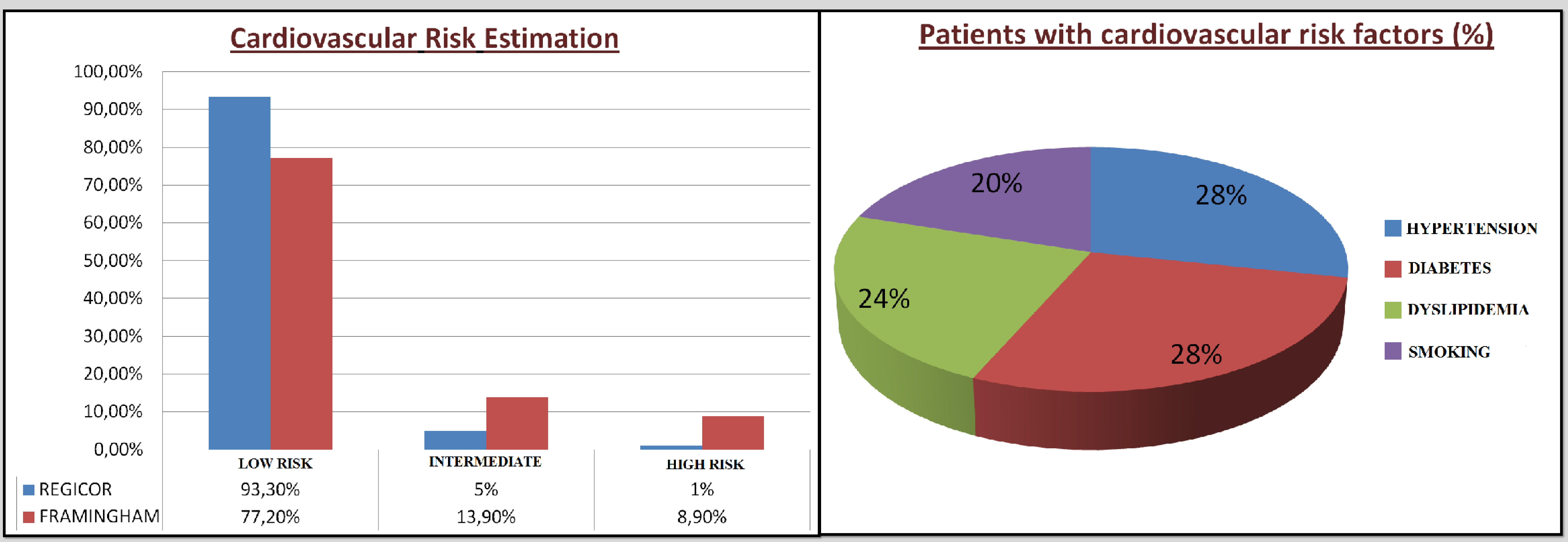

\section{CONCLUSIONS}

In our series, the CVR estimated by Frammigham score was superior to that obtained by REGICOR scale. A positive correlation between both scales was obtained but with a fair agreement. These results support the importance of CVR scales adjusted for each population. 